\title{
Network pharmacology-based identification of key pharmacological pathways of Yin-Huang- Qing-Fei capsule acting on chronic bronchitis
}

This article was published in the following Dove Press journal:

International Journal of COPD

22 December 2016

Number of times this article has been viewed

\author{
Guohua $\mathrm{Yu}^{1,2, *}$ \\ Yanqiong Zhang ${ }^{2, *}$ \\ Weiqiong $\operatorname{Ren}^{3}$ \\ Ling Dong' \\ Junfang $\mathrm{Li}^{2,4}$ \\ Ya Geng ${ }^{2,5}$ \\ Yi Zhang ${ }^{2}$ \\ Defeng $\mathrm{Li}^{2}$ \\ Haiyu $\mathrm{Xu}^{2}$ \\ Hongjun Yang ${ }^{2}$ \\ 'School of Chinese Materia Medica, \\ Beijing University of Chinese \\ Medicine, ${ }^{2}$ Institute of Chinese \\ Materia Medica, China Academy of \\ Chinese Medical Sciences, Beijing, \\ ${ }^{3}$ The First Affiliated Hospital of \\ Hunan University of Traditional \\ Chinese Medicine, Changsha, ${ }^{4}$ School \\ of Chinese Materia Medica, Tianjin \\ University of Traditional Chinese \\ Medicine, Tianjin, ${ }^{5}$ School of Basic \\ Medicine, Shandong University of \\ Chinese Medicine, Jinan, China \\ *These authors contributed equally \\ to this work
}

\begin{abstract}
For decades in China, the Yin-Huang-Qing-Fei capsule (YHQFC) has been widely used in the treatment of chronic bronchitis, with good curative effects. Owing to the complexity of traditional Chinese herbal formulas, the pharmacological mechanism of YHQFC remains unclear. To address this problem, a network pharmacology-based strategy was proposed in this study. At first, the putative target profile of YHQFC was predicted using MedChem Studio, based on structural and functional similarities of all available YHQFC components to the known drugs obtained from the DrugBank database. Then, an interaction network was constructed using links between putative YHQFC targets and known therapeutic targets of chronic bronchitis. Following the calculation of four topological features (degree, betweenness, closeness, and coreness) of each node in the network, 475 major putative targets of YHQFC and their topological importance were identified. In addition, a pathway enrichment analysis based on the Kyoto Encyclopedia of Genes and Genomes pathway database indicated that the major putative targets of YHQFC are significantly associated with various pathways involved in anti-inflammation processes, immune responses, and pathological changes caused by asthma. More interestingly, eight major putative targets of YHQFC (interleukin [IL]-3, IL-4, IL-5, IL-10, IL-13, FCER1G, CCL11, and EPX) were demonstrated to be associated with the inflammatory process that occurs during the progression of asthma. Finally, a molecular docking simulation was performed and the results exhibited that 17 pairs of chemical components and candidate YHQFC targets involved in asthma pathway had strong binding efficiencies. In conclusion, this network pharmacology-based investigation revealed that YHQFC may attenuate the inflammatory reaction of chronic bronchitis by regulating its candidate targets, which may be implicated in the major pathological processes of the asthma pathway.
\end{abstract}

Keywords: traditional Chinese medicine, Yin-Huang-Qing-Fei capsule, chronic bronchitis, network pharmacology, asthma pathway

\section{Introduction}

Chronic bronchitis, a very common disease associated with chronic inflammation, is caused by a variety of etiological factors, such as air pollution, smoking, and pathogen infection. ${ }^{1,2}$ It has been predicted to be the fourth leading cause of death by $2030 .^{3,4}$ There are two stages of episodes for chronic bronchitis: recurrent reaction and acute exacerbation. ${ }^{5}$ The main symptoms of recurrent reaction include cough, asthma, and expectoration, while acute exacerbation is diagnosed when patients present with increased sputum volume, cough, and worsening dyspnea. ${ }^{5}$ Although current therapies, including bronchodilators, anti-tussive agents, steroids, and leukotriene (LT) receptor antagonists, can alleviate some major symptoms of chronic bronchitis, ${ }^{6}$ the treatments
Correspondence: Haiyu Xu; Hongjun Yang

Institute of Chinese Materia Medica, China Academy of Chinese Medical Sciences, Number 16 Nanxiaojie, Dongzhimennei, Beijing 100700, China Tel +86 I37 0l00 469l; $+8613910000292$

Fax +86 640I 44II 2948

Email hy_xu627@I63.com;

hongjun0420@vip.sina.com 
based on these drugs often lead to a series of adverse reactions. ${ }^{7}$ Therefore, it is of great clinical significance to develop more efficient therapeutic strategies to both improve the curative therapy of patients with chronic bronchitis and reduce the incidence of side effects of current treatments. ${ }^{2}$

Traditional Chinese medicine (TCM), as a major component of complementary and alternative medicine remedies, has attracted increasing attention worldwide because of its satisfactory clinical efficacy. ${ }^{8}$ It has also played an important role in preventing people from contracting chronic bronchitis for centuries. ${ }^{9}$ In recent years, the Yin-Huang-Qing-Fei capsule (YHQFC), based on the famous classical formula for the Ma-Xing-Shi-Gan Decoction from the TCM book Treatise on Febrile Diseases (Shang Han Lun), has become a typical formula used to treat chronic bronchitis and has produced satisfactory therapeutic effects clinically. One study reported that the effective rate of treatment for patients with acute exacerbation of chronic obstructive pulmonary disease in the group receiving YHQFC was $97.5 \%$, compared to $85.0 \%$ in the control group. ${ }^{10} \mathrm{YHQFC}$ contains 14 Chinese herbs including Ephedra sinica Stapf (Mi Ma Huang [MMH]), Lepidium apetalum Willd. (Bei Ting Li Zi [BTLZ]), and Semen Armeniacae Amarum (Ku Xing Ren [KXR]). Zhou et $\mathrm{al}^{11}$ identified 54 compounds in YHQFC using the high performance liquid chromatography coupled with quadrupole time-of-flight mass spectrometry method and preliminarily explored the bioactive components of this formula. Additionally, a serum containing YHQFC showed a strong anti-respiratory syncytial virus effect in vitro and was found to inhibit influenza virus replication in cells to a certain degree. ${ }^{12,13}$ Because YHQFC contains a great number of chemical compounds and regulates diverse targets, precisely determining the pharmacological mechanisms of its therapeutic actions and deciphering the relationships between the herbs and diseases are challenges.

Network pharmacology is emerging as a promising strategy, one that is closely related to the application of multiple omics- and systems biology-based technologies. ${ }^{14}$ It is a valuable tool for achieving a holistic view of and comprehensive and systematic insight into the mechanisms of multi-ingredient medicine..$^{15}$ Various molecular networks of complex ingredients and multilevel target-based protein and gene interactions have been constructed for predicting their functions and promoting discovery of active compounds. ${ }^{16}$ Because TCM herbal formulas are considered to be multi-component and multi-target therapeutics, the methodologies of network pharmacology are suitable for pursuing a priori knowledge about the combinations of rules embedded in these formulas. ${ }^{17}$ Thus, the application of network pharmacology to TCM provides new opportunities to understand the interactions between active compounds and relevant targets, which in turn highlight the mechanisms of action. ${ }^{8} 18$ Using this context, the aim of this study was to develop a comprehensive network pharmacology-based approach to investigate the pharmacological mechanisms of YHQFC acting on chronic bronchitis. Figure 1 depicts a flowchart of the experimental procedures of our study.

\section{Materials and methods Ethics}

All experimental procedures were carried out according to the Guideline for Experimentation of the China Academy of Chinese Medical Sciences, and the protocol was reviewed and approved by the Ethics Committee of the institution.

\section{Data preparation}

\section{Construction of the chemical information database of YHQFC}

The chemical components of each herb contained in YHQFC - Ephedra sinica Stapf (MMH), Lepidium apetalum Willd. (BTLZ), Semen Armeniacae Amarum (KXR), Fritillaria thunbergii Miq. (Zhe Bei Mu [ZBM]), Eriobotrya japonica (Thunb.) Lindl. (Pi Pa Ye [PPY]), Gypsum Fibrosum (Sheng Shi Gao [SSG]), Acorus tatarinowii Schott (Shi Chang $\mathrm{Pu}[\mathrm{SCP}]$ ), Isatis indigotica Fort. (Da Qing Ye [DQY]), Artemisia rupestris L. (Xin Jiang Yi Zhi Hao [XJYZH]), Dioscorea nipponica Makino (Chuan Shan Long [CSL]), Schisandra chinensis (Turcz.) Baill. (Wu Wei Zi [WWZ]), Ginkgo biloba L (Yin Xing Ye [YXY]), Citrus aurantium L. (Zhi Shi [ZS]), and Glycyrrhiza uralensis Fisch. (Gan Cao [GC]) - were collected from the Chinese Academy of Sciences chemistry database (http://www.organchem.csdb. $\mathrm{cn} / \mathrm{scdb} / \mathrm{main} / \mathrm{slogin}$.asp, updated on July 20, 2015). This database displays chemical information, including chemical and crystal structures, spectra, reactions, syntheses, and thermo-physical properties. ${ }^{19}$ The available structural information for YHQFC included 29 compounds in MMH, 12 compounds in BTLZ, 7 compounds in KXR, 34 compounds in ZBM, 24 compounds in PPY, 1 compound in $\mathrm{SSG}\left(\right.$ as $\left.\mathrm{CaSO}_{4} \cdot 2 \mathrm{H}_{2} \mathrm{O}\right), 18$ compounds in SCP, 14 compounds in DQY, 35 compounds in XJYZH, 7 compounds in CSL, 89 compounds in WWZ, 55 compounds in YXY, 78 compounds in ZS, and 190 compounds in GC. Detailed information on the constituent compounds of each herb contained in YHQFC is provided in Table S1. The molecular files of all the constituent compounds were downloaded from the ChemSpider database (http://www.chemspider.com/, updated on November 29, 2015) and were saved in .mol format. 


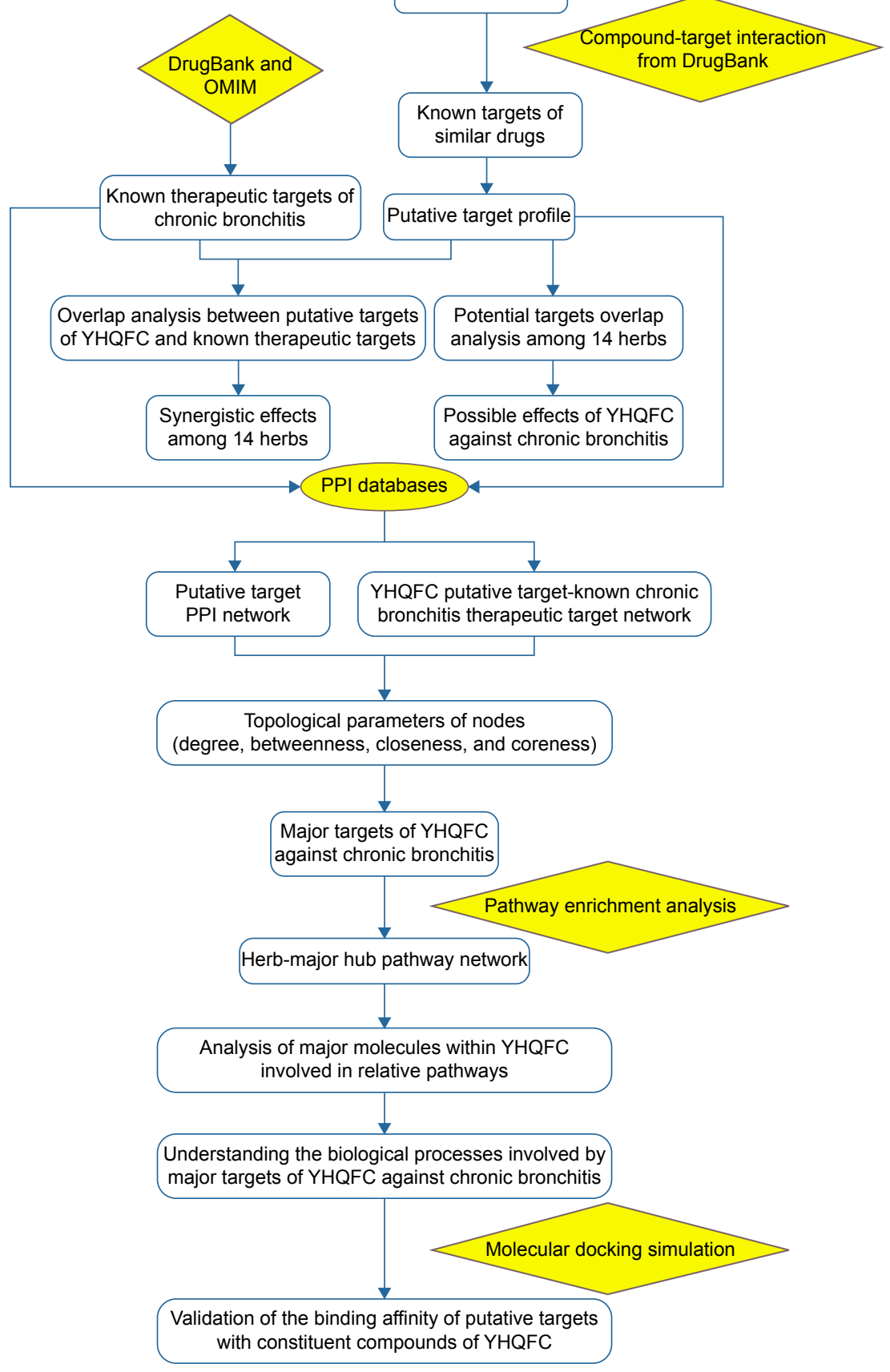

Figure I Whole framework of this study based on network pharmacology for deciphering pharmacological mechanisms of YHQFC acting on chronic bronchitis. Abbreviations: YHQFC, Yin-Huang-Qing-Fei capsule; OMIM, Online Mendelian Inheritance in Man; PPI, protein-protein interaction.

\section{Known therapeutic targets of drugs in the treatment} of chronic bronchitis

The known therapeutic targets of drugs used in the treatment of chronic bronchitis were acquired from two sources: DrugBank $^{20}$ (http://www.drugbank.ca/, version 4.3) and the Online Mendelian Inheritance in Man (OMIM) database ${ }^{21}$ (http://www.omim.org/, updated on April 21, 2016). Using drug-target interactions mentioned in DrugBank, only drugs approved by the Food and Drug Administration (FDA) were selected for the treatment of chronic bronchitis and whose targets are human genes/proteins. In total, we chose 238 known therapeutic targets for treating chronic bronchitis were 
chosen. In addition, the main symptoms of chronic bronchitis, such as "cough," "asthma," and "bronchitis," were used as key words when searching the OMIM database, and the resulting 286 known therapeutic targets were enrolled in this study. Detailed information about these known therapeutic targets is provided in Table S2. After removing redundant entries, 381 known therapeutic targets for the treatment of chronic bronchitis were used for the data analysis.

\section{Prediction of putative targets of YHQFC}

A drug similarity search tool MedChem Studio (version 3.0; Simulations Plus, Inc., Lancaster, CA, USA, 2012), ${ }^{22}$ effective for quick identification of all molecules with structure similarities, was used to identify known drugs that are structurally similar to the chemical components of YHQFC. Then, the therapeutic targets of these known drugs, obtained from the DrugBank database, were considered as putative targets of YHQFC. To improve the reliability of the target prediction results, only drugs with a high structural similarity score $(>0.80$, moderately similar to very similar) were selected.

\section{Protein-protein interaction data}

Protein-protein interaction (PPI) data were derived from eight existing PPI databases, including HAPPI, ${ }^{23}$ Reactome, ${ }^{24}$ OPHID,${ }^{25}$ InAct, ${ }^{26}$ HPRD,${ }^{27}$ MINT, ${ }^{28}$ DIP,${ }^{29}$ and PDZBase. ${ }^{30}$ Detailed information about these PPI databases is provided in Table S3. The differing ID types of the proteins were converted to UniProt IDs.

\section{Network construction and analysis}

Putative YHQFC target-known therapeutic targets of the chronic bronchitis network and putative YHQFC target networks were constructed to understand the associations between the herbs and chemical compounds containing YHQFC, putative targets of YHQFC, and known therapeutic targets of chronic bronchitis. Network visualization was performed using Navigator software (version 2.2.1, Toronto, ON, USA).

\section{Putative YHQFC target-known therapeutic targets of the chronic bronchitis network}

Putative YHQFC target-known therapeutic targets of the chronic bronchitis network were constructed using the links between putative targets of YHQFC and known therapeutic targets of chronic bronchitis. As previously reported, ${ }^{31}$ if the degree of a node is more than twofold the median degree of all the nodes in a network, the node may function as a hub. Subsequently, the hub network was constructed based on the direct interactions among hubs. Moreover, four topological properties, "degree", "betweenness", "closeness", and "coreness", were calculated to screen the putative targets for topological importance. The definitions of the four topological features listed earlier are provided in Supplementary File

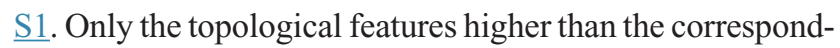
ing median values were identified as the major hubs.

\section{Pathway enrichment analysis}

To clarify the pathways that are involved in putative YHQFC targets, a pathway enrichment analysis was performed using the Database Visualization and Integrated Discovery software $^{32}$ (DAVID, http://david.abcc.ncifcrf.gov/home. jsp, version 6.7) and based on the pathway data obtained from the Kyoto Encyclopedia of Genes and Genomes database $^{33}$ (KEGG, http://www.genome.jp/kegg/, updated on April 18, 2016).

\section{Molecular docking simulation}

To verify the binding affinity of candidate targets to constituent compounds of YHQFC, a molecular docking simulation was performed using the program LibDock implemented in Discovery Studio 2.5 (DS 2.5). All the crystal structures of the candidate targets, including interleukin (IL)-3, IL-5, FCER1G, and EPX, were directly downloaded from the RCSB protein data bank (http://www.pdb.org/, updated on October 21, 2016) and were carefully examined for their resolutions. To evaluate the binding affinity of each candidate target to the corresponding compound, a docking score was calculated by the customizable scoring function of LibDock. The docking scores $>72$ (the median value of all the docking scores) indicated a strong binding affinity of candidate targets to their corresponding compounds.

\section{Results and discussion Putative targets of YHQFC}

A total of 593 constituent compounds of the 14 herbs contained in YHQFC were collected, and 9,199 genes were predicted as the putative targets of YHQFC, including 464 of SCP, 901 of XJYZH, 607 of ZS, 53 of CSL, 1135 of MMH, 491 of PPY, 513 of ZBM, 1535 of YXY, 950 of GC, 197 of SSG, 881 of DQY, 119 of BTLZ, 463 of WWZ, and 890 of KXR. Detailed information about the putative targets of YHQFC is provided in Table S4.

Notably, there were different numbers of common putative targets among the herbs contained in YHQFC, as shown in Table 1, suggesting that these herbs might have several interactions in the course of treatment. Moreover, $>50 \%$ of the putative targets of PPY were common to those of the other herbs, implying a close relationship between PPY and other herbs in the formula. 
Table I Potential target overlaps among 14 herbs of YHQFC

\begin{tabular}{|c|c|c|c|c|c|c|c|c|c|c|c|c|c|c|}
\hline Herbs & $\begin{array}{l}\text { SCP } \\
(464)\end{array}$ & $\begin{array}{l}\text { XJYZH } \\
(901)\end{array}$ & $\begin{array}{l}\text { ZS } \\
(607)\end{array}$ & $\begin{array}{l}\text { CSL } \\
(53)\end{array}$ & $\begin{array}{l}\text { MMH } \\
(I \mid 35)\end{array}$ & $\begin{array}{l}\text { PPY } \\
(491)\end{array}$ & $\begin{array}{l}\text { ZBM } \\
(5 \mid 3)\end{array}$ & $\begin{array}{l}\text { YXY } \\
(1535)\end{array}$ & $\begin{array}{l}\text { GC } \\
(950)\end{array}$ & $\begin{array}{l}\text { SSG } \\
(197)\end{array}$ & $\begin{array}{l}\text { DQY } \\
(88 I)\end{array}$ & $\begin{array}{l}\text { BTLZ } \\
(1 \mid 9)\end{array}$ & $\begin{array}{l}\text { WWZ } \\
\text { (463) }\end{array}$ & $\begin{array}{l}\text { KXR } \\
(890) \\
\end{array}$ \\
\hline SCP (464) & - & 224 & 305 & 21 & 244 & 129 & 139 & 385 & 279 & 57 & 280 & 44 & 293 & 275 \\
\hline XJYZH (90I) & 224 & - & 375 & 25 & 661 & 412 & 294 & 808 & 482 & 69 & 321 & 118 & 299 & 510 \\
\hline ZS (607) & 305 & 375 & - & 14 & 392 & 255 & 230 & 518 & 417 & 70 & 202 & 110 & 393 & 327 \\
\hline CSL (53) & 21 & 25 & 14 & - & 27 & 11 & 53 & 34 & 53 & 8 & 53 & I & 9 & 33 \\
\hline MMH (II35) & 244 & 661 & 392 & 27 & - & 346 & 241 & 908 & 535 & 91 & 514 & 73 & 274 & 405 \\
\hline PPY (49I) & 129 & 412 & 255 & 11 & 346 & - & 190 & 426 & 287 & 48 & 165 & 112 & 219 & 270 \\
\hline ZBM (5|3) & 139 & 294 & 230 & 53 & $24 I$ & 190 & - & 443 & 297 & 54 & 231 & 112 & 178 & 348 \\
\hline YXY (I535) & 385 & 808 & 518 & 34 & 908 & 426 & 443 & - & 719 & 118 & 622 & 119 & 418 & 690 \\
\hline GC (950) & 279 & 482 & 417 & 53 & 535 & 287 & 297 & 719 & - & 88 & 330 & 104 & 309 & 451 \\
\hline SSG (197) & 57 & 69 & 70 & 8 & 91 & 48 & 54 & 118 & 88 & - & 44 & 13 & 57 & 64 \\
\hline DQY (88I) & 280 & 321 & 202 & 53 & 514 & 165 & 231 & 622 & 330 & 44 & - & 50 & 163 & 315 \\
\hline BTLZ (II9) & 44 & 118 & 110 & I & 73 & 112 & 112 & 119 & 104 & 13 & 50 & - & 107 & 115 \\
\hline WWZ (463) & 293 & 299 & 393 & 9 & 274 & 219 & 178 & 418 & 309 & 57 & 163 & 107 & - & 251 \\
\hline KXR (890) & 275 & 510 & 327 & 33 & 405 & 270 & 348 & 690 & 451 & 64 & 315 & 115 & 251 & - \\
\hline
\end{tabular}

Note: Numbers shown within parentheses show total putative targets of each herb.

Abbreviations: YHQFC, Yin-Huang-Qing-Fei capsule; SCP, Shi Chang Pu (Acorus tatarinowii Schott); XJYZH, Xin Jiang Yi Zhi Hao (Artemisia rupestris L.); ZS, Zhi Shi (Citrus aurantium L.); CSL, Chuan Shan Long (Dioscorea nipponica Makino); MMH, Mi Ma Huang (Ephedra sinica Stapf); PPY, Pi Pa Ye (Eriobotrya japonica (Thunb.) Lindl.); ZBM, Zhe Bei Mu (Fritillaria thunbergii Miq.); YXY, Yin Xing Ye (Ginkgo biloba L); GC, Gan Cao (Glycyrrhiza uralensis Fisch.); SSG, Sheng Shi Gao (Gypsum Fibrosum); DQY, Da Qing Ye (Isatis indigotica Fort.); BTLZ, Bei Ting Li Zi (Lepidium apetalum Willd.); WWZ, Wu Wei Zi (Schisandra chinensis [Turcz.] Baill.); KXR, Ku Xing Ren (Semen Armeniacae Amarum).

\section{Pharmacological mechanisms of YHQFC acting on chronic bronchitis}

The putative YHQFC target-known therapeutic target of the chronic bronchitis network consisted of 10,828 nodes and 73,659 edges. Using the twofold median value of node degree (4) in this network as a cutoff point, 3,667 nodes were identified as hubs. Detailed information on these hubs is provided in Table $\mathrm{S} 5$.

Following the construction of a hub interaction network and the calculation of the four topological features, "degree", "betweenness", "closeness", and "coreness", of each hub, 475 major hubs, including 395 putative YHQFC targets and 80 known therapeutic targets of chronic bronchitis, were screened; detailed information on these targets is provided in Table S6. To investigate the biological functions of these major hubs, a pathway enrichment analysis was performed. Consequently, the major hubs were significantly associated with various physiological processes, including inflammation processes and immune suppression (such as asthma pathways, cytokine-cytokine receptor interactions, and T cell receptor signaling pathways), vascular smooth muscle contraction, and neuroactive ligand-receptor interaction (Table 2). Cytokines are soluble mediators (polypeptides) that act as messengers for the immune system, among other biologic functions, and are important for leukocyte growth and differentiation, as well as activation and migration. The overexpression of inflammatory cytokines or decreased levels of anti-inflammatory cytokines can lead to inflammatory disorders. ${ }^{34}$ In human beings, allergic asthma is primarily initiated by a type I hypersensitivity reaction, which represents increased susceptibility to producing immunoglobulin E (IgE) in response to external allergens, the secretion and differentiation of which are dependent on $\mathrm{CD}^{+}$helper $\mathrm{T}$ cells (Th2 type). ${ }^{35,36}$ Activated Th2 cells may produce a number of cytokines (eg, IL-3, IL-4, IL-5, and IL-13), which in turn play roles in various processes, such as promoting production of IgE cells by B cells (IL-4 and IL-13), growth of mast cells (IL-4), and survival of eosinophils (IL-3 and IL-5). ${ }^{37}$ IL-4 and IL-13 also stimulate epithelial cells to produce transforming growth factor alpha, which may cause mucosal metaplasia and fibroblast proliferation..$^{38}$ Proinflammatory mediators, including tumor necrosis factors and granulocyte macrophage colony-stimulating factors, stimulate the expression of vascular adhesion molecules on endothelial cells, which results in an increased influx of inflammatory leukocytes into the

Table 2 Main KEGG pathways significantly relating to major hubs

\begin{tabular}{lll}
\hline KEGG pathways & Counts & P-value \\
\hline hsa04664:Fc epsilon RI signaling pathway & 34 & $2.36 \times 10^{-16}$ \\
hsa04662:B cell receptor signaling pathway & 31 & $3.96 \times 10^{-14}$ \\
hsa04660:T cell receptor signaling pathway & 34 & $1.41 \times 10^{-11}$ \\
hsa04620:Toll-like receptor signaling pathway & 29 & $6.16 \times 10^{-9}$ \\
hsa04080:Neuroactive ligand-receptor interaction & 46 & $8.02 \times 10^{-7}$ \\
hsa0462I:NOD-like receptor signaling pathway & 19 & $1.62 \times 10^{-6}$ \\
hsa04270:Vascular smooth muscle contraction & 26 & $3.58 \times 10^{-6}$ \\
hsa04630:Jak-STAT signaling pathway & 31 & $8.62 \times 10^{-6}$ \\
hsa04650:Natural killer cell-mediated cytotoxicity & 27 & $2.89 \times 10^{-5}$ \\
hsa04060:Cytokine-cytokine receptor interaction & 36 & 0.003305 \\
hsa053 I0:Asthma & 8 & 0.008380 \\
hsa05I30:Pathogenic Escherichia coli infection & 10 & 0.045010 \\
\hline Abbreviation: KEGG, Kyoto Encyclopeda of Genes and
\end{tabular}

Abbreviation: KEGG, Kyoto Encyclopedia of Genes and Genomes. 
bronchial tree. ${ }^{39}$ The pathology of inflammatory processes in chronic airway diseases such as chronic bronchitis is directly associated with increased generation of reactive oxygen species (ROS) in the lungs. ${ }^{40}$ Various enzymatic and nonenzymatic antioxidants are available in the lungs to counteract ROS-mediated damage, which may lead to increased vascular permeability, mucus hypersecretion, smooth muscle contraction, and epithelial shedding. ${ }^{41-45}$ The regulation of vascular smooth muscle contractions contributes to the control of blood pressure and airway remodeling. ${ }^{46,47}$ It is reported that increase in the local concentration of inflammatory neuropeptides may stimulate bronchial fibers, which rapidly adapts receptors and provokes the cough reflex. ${ }^{48}$ The neuroactive ligand-receptor pathway includes many G-protein-coupled receptors, which are often located on the cell surface and involved in the transduction of an endogenous signal into an intracellular response. ${ }^{49} \mathrm{G}$-protein-coupled receptors regulate vital processes by controlling the expression and function of seven transmembrane receptors, which play a particularly important role in some widespread neurological disorders. ${ }^{19}$ For example, the activation of the A1 adenosine receptor in allergic rabbit airway smooth muscle causes the production of inositol 1,4,5-trisphosphate [Ins $(1,4,5) \mathrm{P} 3]$ via a pertussis toxin-sensitive $\mathrm{G}$ protein-coupled phospholipase C. This signaling mechanism may be involved in the generation of contractile responses. It is hypothesized that this process may contribute to adenosine-induced bronchoconstriction in allergic asthma. ${ }^{50}$

A herb-major hub-pathway network was constructed to visualize the relationships among herbs, major targets, and disease (Figure 2). This network illustrated that the major putative targets of YHQFC are mostly involved in pathways related to anti-inflammation processes and the immune system, which may be crucial elements during the attenuation of chronic bronchitis. Interestingly, we also found that YHQFC may exert its therapeutic effects on chronic bronchitis by regulating the pathways related to the contraction of airway vascular smooth muscle. The relevant nerve center regulation induced by the major putative targets of YHQFC was another pathway to calm cough and asthma.

\section{YHQFC attenuates the pathological changes of chronic bronchitis via regulating the asthma pathway}

Four major putative targets of YHQFC (IL-3, IL-5, the FceRI receptors [FCER1G], and eosinophil peroxidase [EPX]) and four known therapeutic targets of chronic bronchitis (IL-4, IL-10, IL-13, and a member of the eotaxin subfamily of CC

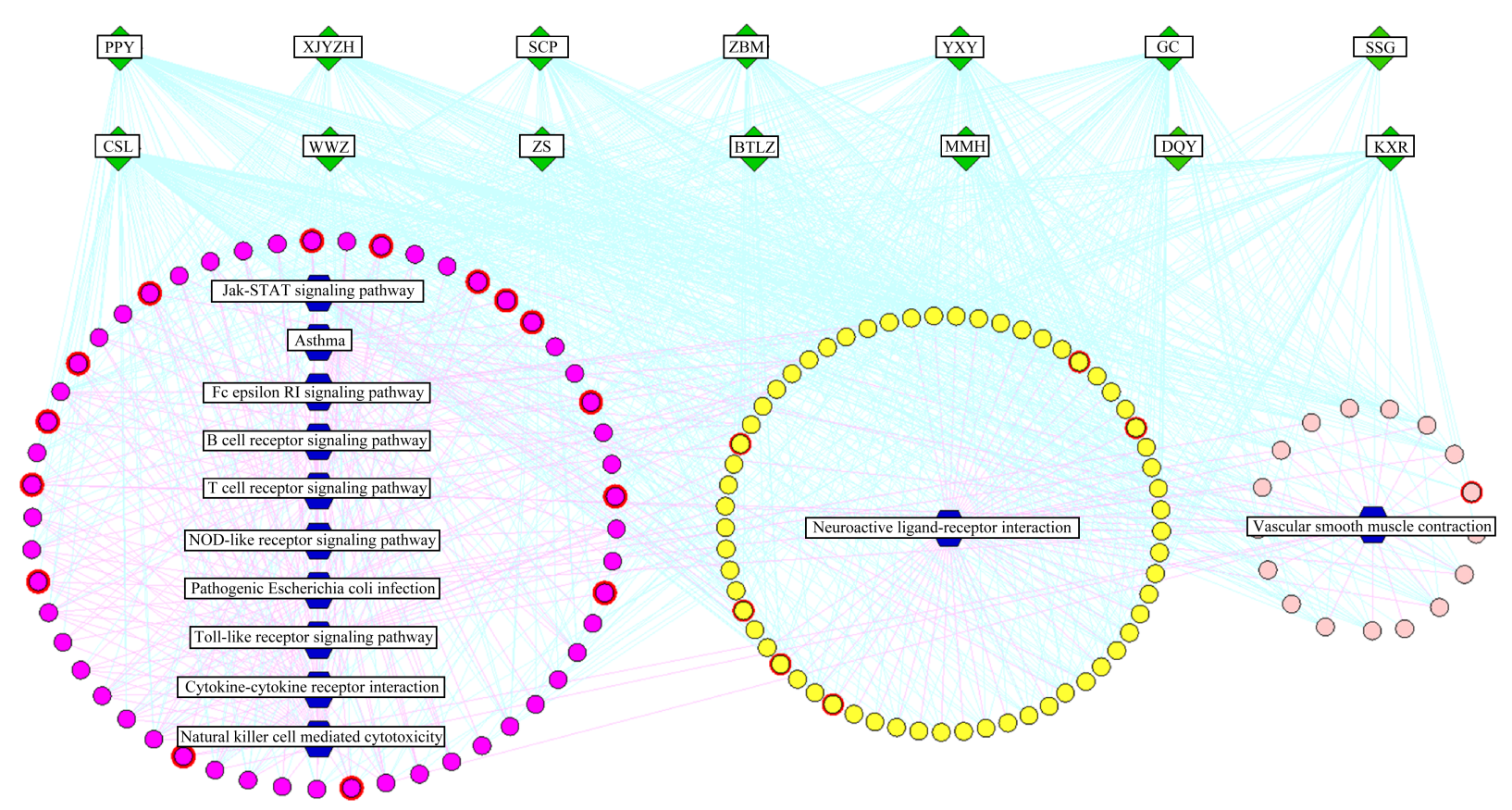

Figure 2 Herb-major hub-pathway network.

Notes: Green diamonds represent each herb in YHQFC; round nodes represent putative targets of constituent compounds of YHQFC; nodes marked with red rings represent known therapeutic targets in the treatment of chronic bronchitis. Blue hexagons represent main pathways from enrichment analysis of major targets. Edges represent interactions among herbs, putative targets, and known therapeutic targets for the treatment of chronic bronchitis.

Abbreviations: YHQFC, Yin-Huang-Qing-Fei capsule; SCP, Shi Chang Pu (Acorus tatarinowii Schott); XJYZH, Xin Jiang Yi Zhi Hao (Artemisia rupestris L.); ZS, Zhi Shi (Citrus aurantium L.); CSL, Chuan Shan Long (Dioscorea nipponica Makino); MMH, Mi Ma Huang (Ephedra sinica Stapf); PPY, Pi Pa Ye (Eriobotrya japonica (Thunb.) Lindl.); ZBM, Zhe Bei Mu (Fritillaria thunbergii Miq.); YXY, Yin Xing Ye (Ginkgo biloba L); GC, Gan Cao (Glycyrrhiza uralensis Fisch.); SSG, Sheng Shi Gao (Gypsum Fibrosum); DQY, Da Qing Ye (Isatis indigotica Fort.); BTLZ, Bei Ting Li Zi (Lepidium apetalum Willd.); WWZ, Wu Wei Zi (Schisandra chinensis [Turcz.] Baill.); KXR, Ku Xing Ren (Semen Armeniacae Amarum). 
chemokines [CCL11]) were demonstrated to participate in the asthma pathway, which has been shown to be involved in the occurrence and progression of chronic bronchitis. As shown in Figure 3, the asthma pathway is mainly composed of inflammatory processes. According to the clusters of major hubs contained in the asthma pathway, YHQFC may be of vital importance for regulating these cytokines and chemokines.

Among the major putative targets of YHQFC, IL-4 and IL-13 both play important roles in the class switching of $\mathrm{B}$ cells to IgE synthesis, as well as in the recruitment of mast cells, while IL-4 alone is crucial for maintaining the Th2 cell phenotype. Several animal studies have shown that blocking the production or inhibiting the effects of IL-4 has profound effects on the allergic phenotype ${ }^{51}$ It is well established that the crosslinking of FceRI receptors by allergen-bound IgE induces the release of inflammatory mediators (eg, histamine), which, in turn, causes the immediate symptoms of inflammatory reaction within $30 \mathrm{~min}^{52}$ The suppression of FCER1G and down-regulation of the expression of FceRI receptors may have effects on relieving the immediate reaction of chronic bronchitis. IL-10 is the main inhibitory cytokine produced by regulatory $\mathrm{T}\left(\mathrm{T}_{\mathrm{Reg}}\right)$ cells that inhibits the expression of many proinflammatory cytokines and chemokines, as well as proinflammatory enzymes. ${ }^{53}$ It has been reported that IL-10-deficient mice had increased airway inflammation after allergen challenges. ${ }^{54}$ The presence of IL-13, as well as epidermal, neural, vascular, and fibroblast growth factors, leads to mucous metaplasia and remodeling of the airway walls. ${ }^{55}$ The numerous functions of IL-13 in regulating IgE production, eosinophilic inflammation, airway smooth muscle hyperplasia, the induction of goblet cell hyperplasia with mucus production, and the recruitment of monocytes, macrophages, and $\mathrm{T}$ cells into the airway spaces make it a key therapeutic target in chronic bronchitis. ${ }^{56}$ Selective blocking of IL-13 has been achieved in mice using a soluble form of IL-13R $\alpha 2$, which has a high affinity with IL-13, and has led to the reversal of airway hyperresponsiveness and mucus production in allergen-exposed sensitized mice. ${ }^{57}$ IL-3 and IL- 5 can be released by Th 2 cells and mast cells when they are stimulated..$^{58,59}$ The function of IL-3 and IL-5 is to promote the maturation of eosinophils, and it is evident that the activation of eosinophils can lead to the production of ROS, including EPX, which are implicated in protein oxidation in the bronchus airway and the late asthmatic response. ${ }^{60-62} \mathrm{CCL} 11$ (eotaxin) is a member of the eotaxin subfamily of CC chemokines and is a potent chemoattractant for eosinophils. ${ }^{63,64}$ It acts predominantly through the eotaxin receptor, CCR3, which has been identified on eosinophils, mast cells, Th2 cells, and airway epithelial cells.$^{65}$ It is evident that CCL11 can be upregulated in a variety of diseases associated with eosinophilic infiltrates,

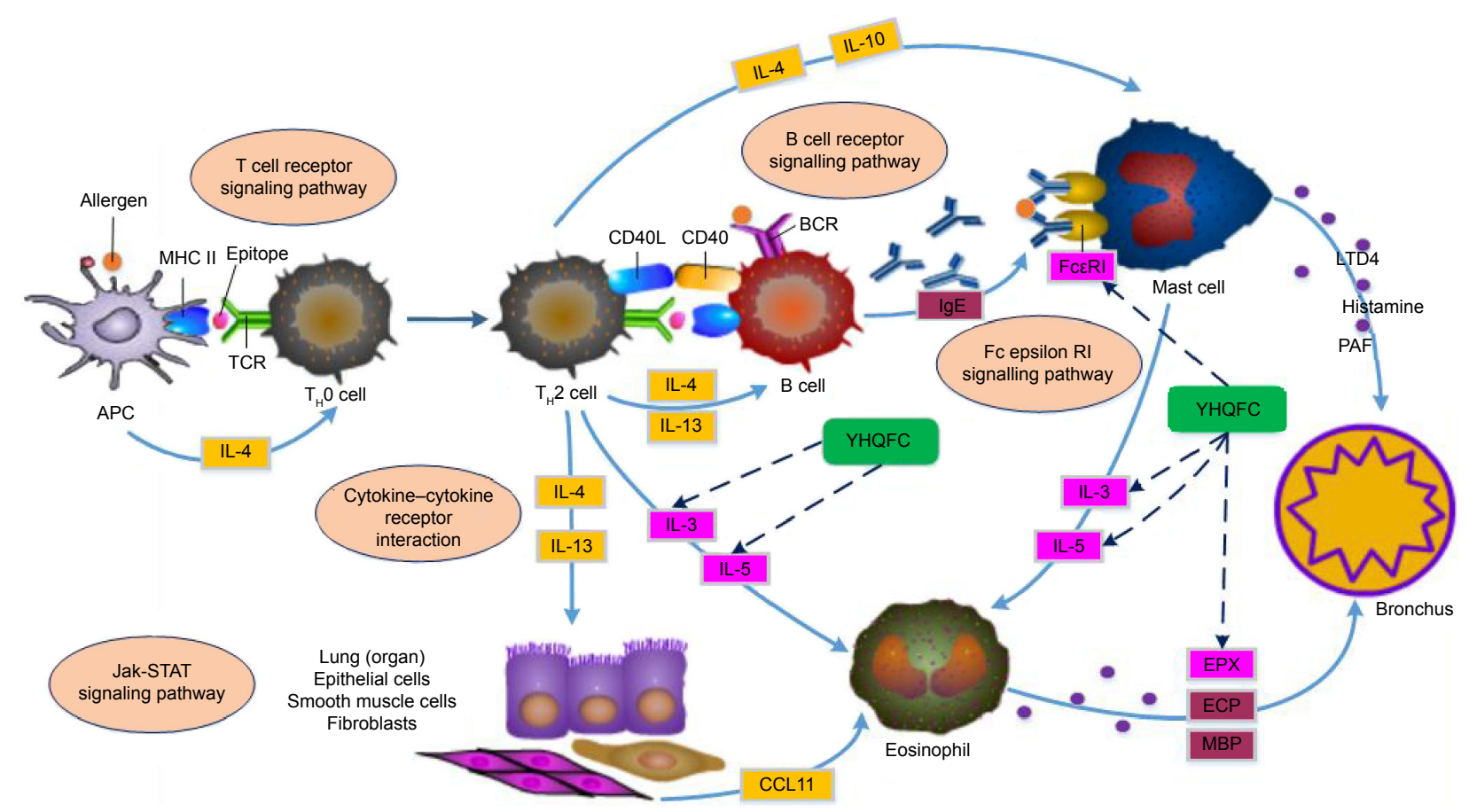

Figure 3 Illustration of the asthma pathway induced by the major putative YHQFC targets and known therapeutic targets of chronic bronchitis.

Abbreviations: YHQFC, Yin-Huang-Qing-Fei capsule; IL, interleukin; APC, antigen-presenting cell; MHC, major histocompatibility complex; Th, T helper; TCR, T cell receptor; BCR, B cell receptor; IgE, immunoglobulin E; LTD4, leukotriene D4; PAF, platelet-activating factor; ECP, eosinophil cationic protein; MBP, myelin basic protein. 
including chronic bronchitis. ${ }^{66}$ Down-regulation of CCL11 and CCR3 receptor antagonists may be useful for blocking eosinophil entry and degranulation in chronic bronchitis. ${ }^{67}$

Importantly, among the four major putative targets involved in the asthma pathway, IL-3 is the putative target of 2-methyl-1,3,6-trihydroxyanthraquinone (constituent compound from GC) and angeloylgomisin Q (constituent compound from WWZ). FCER1G is the putative target of phenylalanine (constituent compound from YXY), mandelic acid (constituent compound from KXR), choline (constituent compound from ZBM), and isolobelanine (constituent compound from GC). EPX is the putative target of euasarone (constituent compound from SCP) and $N$-methyltyramine (constituent compound from ZS). IL-5 is the putative target of multiple constituent compounds, including naringenin (from ZS) and isoginkgetin (from YXY). These data may provide more evidence for the identification of the active compounds of YHQFC that act on chronic bronchitis.

\section{Molecular docking validation}

It is evident that the computational docking simulation is an invaluable tool in drug discovery and design. This technique is structure-based and could help researchers discover the interactions between the components of TCM and network targets. ${ }^{68}$ DS LibDock is an efficient and high-throughput module in which the ligand will be aligned in the active site. LibDock consensus score can be used to evaluate the binding efficiency of a ligand, which is based on the geometry of a candidate ligand docked into a target receptor structure using empirical functions. ${ }^{69}$ In this study, a docking score $>72$ indicated a strong binding capacity between the constituent compounds of YHQFC and the candidate targets. And 17 pairs of compound-candidate effector molecule interactions were of strong binding affinity. Detailed information about the docking scores of the candidate targets binding to their corresponding compounds contained in YHQFC is described in Table S7. To investigate the regulation of these compounds on the corresponding candidate targets, known drugs were collected with similar structures to these compounds and their experimentally validated interactions with the corresponding candidate targets using Medchem Studio. As drugs with similar structures often possess similar functions, the regulatory effects of compounds on candidate effectors of YHQFC may be similar to those of the corresponding similar known drugs. As a result, 29 pairs of compound-candidate effector molecule interactions have positive effects and 4 pairs have negative effects. Detailed information about the regulatory effects of similar known drugs on candidate targets of YHQFC is described in Table S8. These findings should be validated by further biological experiments.

\section{Conclusion}

Chinese herbal formulas are one of the most important types of complementary and alternative medicine and contribute greatly to respiratory disease therapy. This study proposed and applied network pharmacology-based analysis to suggest that YHQFC may attenuate the inflammatory response of chronic bronchitis by regulating its candidate targets implicated into the asthma pathway. Further experimental validations of these prediction results are required in our future studies.

\section{Acknowledgments}

This study was supported by the Beijing Nova program (Z1511000003150126). The authors wish to acknowledge Dr Shihuan Tang for performing the molecular docking simulation for our research.

\section{Disclosure}

The authors report no conflicts of interest in this work.

\section{References}

1. Miravitlles M. Moxifloxacin in the management of exacerbations of chronic bronchitis and COPD. Int J Chron Obstruct Pulmon Dis. 2007; 2(3):191-204.

2. Ko HJ, Oh SK, Jin JH, Son KH, Kim HP. Inhibition of experimental systemic inflammation (septic inflammation) and chronic bronchitis by new phytoformula BL containing Broussonetia papyrifera and Lonicera japonica. Biomol Ther (Seoul). 2013;21(1):66-71.

3. Mathers CD, Loncar D. Projections of global mortality and burden of disease from 2002 to 2030. PLoS Med. 2006;3(11):442-442.

4. Hunter DJ, Reddy KS. Noncommunicable diseases. NEngl J Med. 2013; 369(14):1336-1343.

5. Liu S, Shergis J, Chen X, et al. Chinese herbal medicine (Weijing decoction) combined with pharmacotherapy for the treatment of acute exacerbations of chronic obstructive pulmonary disease. Evid Based Complement Alternat Med. 2014;2014(8):1-12.

6. Jeffery PK. Remodeling in asthma and chronic obstructive lung disease. Am J Respir Crit Care Med. 2001;164(supp1 2):S28-S38.

7. Liu W, Jiang H, Zhang R, et al. Efficacy and safety of Qing-FengGan-Ke granules in patients with postinfectious cough: study protocol of a novel-design phase III placebo-controlled, double-blind randomized trial. BMC Complement Altern Med. 2015;15(1):1-11.

8. Tao W, Xu X, Wang X, et al. Network pharmacology-based prediction of the active ingredients and potential targets of Chinese herbal radix curcumae formula for application to cardiovascular disease. J Ethnopharmacol. 2013;145(1):1-10.

9. Li XM. Traditional Chinese herbal remedies for asthma and food allergy. J Allergy Clin Immunol. 2007;120(1):25-31.

10. Ye HF, Xie HB. Effect and clinical observation of Yinghuang Qingfei capsules on the hemorrheology of patients with acute exacerbation of chronic obstructive pulmonary disease. China Pharm. 2015;2015(11): 1536-1538.

11. Wang D, Cai T, Wu ZJ, et al. LC-ESI-MS/MS analysis of chemical constituents in Yinhuang Qingfei capsule. China Meas Test. 2016;42(03): $36-40$. 
12. Li XL, Lu FG, Yi DF, et al. Experimental research on the function of anti-influenza virus in vitro of Yinhuang Qingfei capsules containing serum. Chin Arch Tradit Chin Med. 2015;2015(05):1107-1109.

13. Peng Z, Lu FG, Qu JY, et al. Experimental research of Yinhuang Qingfei capsules containing serum on the respiratory syncytial virus in vitro. J Hunan Univ Chin Med. 2016;36(02):40-43.

14. Liang X, Li H, Li S. A novel network pharmacology approach to analyse traditional herbal formulae: the Liu-Wei-Di-Huang pill as a case study. Mol Biosyst. 2014;10(5):1014-1022.

15. Wang S, Tong Y, Ng TB, et al. Network pharmacological identification of active compounds and potential actions of Erxian decoction in alleviating menopause-related symptoms. Chin Med. 2015; 10(1):1-12.

16. Zhang GB, Li QY, Chen QL, et al. Network pharmacology: a new approach for Chinese herbal medicine research. Evid Based Complement Alternat Med. 2013;2013(8):1-9.

17. Li S, Zhang B, Jiang D, Wei Y, Zhang N. Herb network construction and co-module analysis for uncovering the combination rule of traditional Chinese herbal formulae. BMC Bioinformatics. 2010;11(suppl 11): S1-S12.

18. Zhao F, Guochun L, Yang Y, Shi L, Xu L, Yin L. A network pharmacology approach to determine active ingredients and rationality of herb combinations of modified-Simiaowan for treatment of gout. J Ethnopharmacol. 2015;168:1-16.

19. Zhang YQ, Wang SS, Zhu WL, et al. Deciphering the pharmacological mechanism of the Chinese formula Huanglian-Jie-Du decoction in the treatment of ischemic stroke using a systems biology-based strategy. Acta Pharmacol Sin. 2015;36(6):724-733.

20. Sheng S, Wang J, Wang L, et al. Network pharmacology analyses of the antithrombotic pharmacological mechanism of Fufang Xueshuantong capsule with experimental support using disseminated intravascular coagulation rats. J Ethnopharmacol. 2014;154(3):735-744.

21. Hamosh A, Scott AF, Amberger JS, Bocchini CA, McKusick VA. Online Mendelian inheritance in man (OMIM), a knowledgebase of human genes and genetic disorders. Nucleic Acids Res. 2005;33(suppl 1): S514-S517.

22. Xu H, Tao Y, Lu P, et al. A computational drug-target network for Yuanhu Zhitong prescription. Evid Based Complement Alternat Med. 2013;2013(1):70-75.

23. Chen JY, Mamidipalli S, Huan T. HAPPI: an online database of comprehensive human annotated and predicted protein interactions. BMC Genomics. 2009;10(suppl 1):S1-S11.

24. D'Eustachio P. Reactome knowledgebase of human biological pathways and processes. Bioinform Comp Proteomics. 2009;37(37):49-61.

25. Brown KR, Jurisica I. Online predicted human interaction database. Bioinformatics. 2005;21(9):2076-2082.

26. Kerrien S, Aranda B, Breuza L, et al. The IntAct molecular interaction database in 2012. Nucleic Acids Res. 2012;40(21):841-846.

27. Prasad TK, Kandasamy K, Pandey A. Human protein reference database and human proteinpedia as discovery tools for systems biology. Methods Mol Biol. 2009;577(577):67-79.

28. Ceol A, Aryamontri AC, Licata L, et al. MINT, the molecular interaction database: 2009 update. Nucleic Acids Res. 2009;38:532-539.

29. Lehne B, Schlitt T. Protein-protein interaction databases: keeping up with growing interactomes. Hum Genomics. 2009;3(3):291-297.

30. Beuming T, Skrabanek L, Niv MY, et al. PDZBase: a protein-protein interaction database for PDZ-domains. Bioinformatics. 2005;21(6): $827-828$.

31. Li S, Zhang ZQ, Wu LJ, Zhang XG, Li YD, Wang YY. Understanding ZHENG in traditional Chinese medicine in the context of neuroendocrine-immune network. IET Syst Biol. 2007;1(1):51-60.

32. Dennis G Jr, Sherman BT, Hosack DA, et al. DAVID: database for annotation, visualization, and integrated discovery. Genome Biol. 2003; 4(5):3-3.

33. Kanehisa M, Goto S. KEGG: Kyoto encyclopedia of genes and genomes. Nucleic Acids Res. 2000;28(1):27-30.

34. Steinke JW, Borish L. Cytokines and chemokines. J Allergy Clin Immunol. 2006;117(2):441-445.
35. Kumar V, Abbas AK, Aster JC, et al. Robbins and Cotran Pathologic Basis of Disease. 4th ed. Pennsylvania: Elsevier Saunders; 2005.

36. PlattsMills TA. The role of immunoglobulin E in allergy and asthma. Am J Respir Crit Care Med. 2001;164(8 pt 2):1-5.

37. Bjermer L, Diamant Z. Current and emerging nonsteroidal antiinflammatory therapies targeting specific mechanisms in asthma and allergy. Treat Respir Med. 2004;3(3):235-246.

38. Nakae S, Ho LH, Yu M, et al. Mast cell-derived TNF contributes to airway hyperreactivity, inflammation, and $\mathrm{TH} 2$ cytokine production in an asthma model in mice. J Allergy Clin Immunol. 2007;120(1):48-55.

39. Zeiger RS, Schatz M, Li Q, et al. High blood eosinophil count is a risk factor for future asthma exacerbations in adult persistent asthma. J Allergy Clin Immunol Pract. 2014;2(6):741-750.

40. Shokry DM, Eltarahony SA. Oxidant-antioxidant balance in childhood asthma. Egypt J Pediatr Allergy Immunol. 2013;11(1):35-40.

41. Lee IT, Yang CM. Role of NADPH oxidase/ROS in pro-inflammatory mediators-induced airway and pulmonary diseases. Biochem Pharmacol. 2012;84(5):581-590.

42. Reszka KJ, Sallans L, Macha S, et al. Airway peroxidases catalyze nitration of the \{beta 2 -agonist salbutamol and decrease its pharmacological activity. J Pharmacol Exp Ther. 2011;336(2):440-449.

43. Dröge W. Free radicals in the physiological control of cell function. Physiol Rev. 2002;82(1):47-95.

44. Evans P, Halliwell B. Micronutrients: oxidant/antioxidant status. Br J Nutr. 2001;85(supp1 2):S67-S74.

45. Chaudière J, Ferrari-Iliou R. Intracellular antioxidants: from chemical to biochemical mechanisms. Food Chem Toxicol. 1999;37(9-10): 949-962.

46. Brozovich FV, Nicholson CJ, Degen CV, Gao YZ, Aggarwal M, Morgan KG. Mechanisms of vascular smooth muscle contraction and the basis for pharmacologic treatment of smooth muscle disorders. Pharmacol Rev. 2016;68(2):476-532.

47. Nadeem A, Siddiqui N, Alharbi NO, Alharbi MM. Airway and systemic oxidant-antioxidant dysregulation in asthma: a possible scenario of oxidants spill over from lung into blood. Pulm Pharmacol Ther. 2014;29(1):31-40.

48. Franova S, Nosal'Ova G. ACE-inhibitors and defence reflexes of the airways. Acta Physiol Hung. 1997;85(4):359-366.

49. Gunawardhana LP, Gibson PG, Simpson JL, Benton MC, Lea RA, Baines KJ. Characteristic DNA methylation profiles in peripheral blood monocytes are associated with inflammatory phenotypes of asthma. Epigenetics. 2014;9(9):1302-1316.

50. Abebe W, Mustafa SJ. A1 adenosine receptor-mediated Ins $(1,4,5)$ P3 generation in allergic rabbit airway smooth muscle. Am J Physiol. 1998;275(5):990-997.

51. Holgate ST, Polosa R. Treatment strategies for allergy and asthma. Nat Rev Immunol. 2008;8(3):218-230.

52. Valenta R. The future of antigen-specific immunotherapy of allergy. Nat Rev Immunol. 2002;2(6):446-453.

53. Wu K, Bi Y, Sun K, Wang C. IL-10-producing type 1 regulatory T cells and allergy. Cell Mol Immunol. 2007;4(4):269-275.

54. Grünig G, Corry DB, Leach MW, Seymour BW, Kurup VP, Rennick DM. Interleukin-10 is a natural suppressor of cytokine production and inflammation in a murine model of allergic bronchopulmonary aspergillosis. J Exp Med. 1997;185(6):1089-1099.

55. Nelson HS, Davies DE, Wicks J, Powell RM, Puddicombe SM, Holgate ST. Airway remodeling in asthma: new insights. $J$ Allergy Clin Immunol. 2003;111(2):215-225.

56. Wynn TA. IL-13 effector functions. Annu Rev Immunol. 2003;21(1): 425-456.

57. Grunig G, Warnock M, Wakil AE, et al. Requirement for IL-13 independently of IL-4 in experimental asthma. Science. 1998;282(5397): 2261-2263.

58. Galli SJ, KalesnikoffJ, Grimbaldeston MA, Piliponsky AM, Williams CM, Tsai M. Mast cells as "tunable" effector and immunoregulatory cells: recent advances. Annu Rev Immunol. 2005;23:749-786.

59. Renauld J-C. New insights into the role of cytokines in asthma. J Clin Pathol. 2001;54(8):577-589. 
60. Cieslewicz G, Tomkinson A, Adler A, et al. The late, but not early, asthmatic response is dependent on IL-5 and correlates with eosinophil infiltration. J Clin Invest. 1999;104(3):301-308.

61. Dombrowicz D, Capron M. Eosinophils, allergy and parasites. Curr Opin Immunol. 2001;13(6):716-720.

62. Wu W, Samoszuk MK, Comhair SA, et al. Eosinophils generate brominating oxidants in allergen-induced asthma. J Clin Invest. 2000; 105(10):1455-1463.

63. Van CE, Van DJ, Opdenakker G. The MCP/eotaxin subfamily of CC chemokines. Cytokine Growth Factor Rev. 1999;10(1):61-86.

64. Ponath PD, Qin S, Ringler DJ, et al. Cloning of the human eosinophil chemoattractant, eotaxin. Expression, receptor binding, and functional properties suggest a mechanism for the selective recruitment of eosinophils. J Clin Invest. 1996;97(3):604-612.

65. Kodali RB, Kim WJ, Galaria II, et al. CCL11 (eotaxin) induces CCR3dependent smooth muscle cell migration. Arterioscler Thromb Vasc Biol. 2004;24(7):1211-1216.
66. Rankin SM, Conroy DM, Williams TJ. Eotaxin and eosinophil recruitment: implications for human disease. Mol Med Today. 2000;6(1):20-27.

67. Heath H, Qin S, Rao P, et al. Chemokine receptor usage by human eosinophils. The importance of CCR3 demonstrated using an antagonistic monoclonal antibody. J Clin Invest. 1997;99(2):178-184.

68. Zhang G, Li Q, Chen Q, Su S. Network pharmacology: a new approach for Chinese herbal medicine research. Evid Based Complement Alternat Med. 2013;2013(8):621423-621423.

69. Ai G, Tian C, Deng D, et al. A combination of 2D similarity search, pharmacophore, and molecular docking techniques for the identification of vascular endothelial growth factor receptor-2 inhibitors. Anticancer Drugs. 2015;26(4):399-409.

\section{Publish your work in this journal}

The International Journal of COPD is an international, peer-reviewed journal of therapeutics and pharmacology focusing on concise rapid reporting of clinical studies and reviews in COPD. Special focus is given to the pathophysiological processes underlying the disease, intervention programs, patient focused education, and self management protocols.

\section{Dovepress}

This journal is indexed on PubMed Central, MedLine and CAS. The manuscript management system is completely online and includes a very quick and fair peer-review system, which is all easy to use. Visit $\mathrm{http}: / / \mathrm{www}$.dovepress.com/testimonials.php to read real quotes from published authors.

Submit your manuscript here: http://www.dovepress.com/international-journal-of-chronic-obstructive-pulmonary-disease-journal 